Meta

Journal des traducteurs

Translators' Journal

\title{
Lexique anglais-français des sous-systèmes des satellites de télécommunications
}

\section{Tanja Collet}

Volume 37, numéro 3, septembre 1992

URI : https://id.erudit.org/iderudit/002349ar

DOI : https://doi.org/10.7202/002349ar

Aller au sommaire du numéro

Éditeur(s)

Les Presses de l'Université de Montréal

ISSN

0026-0452 (imprimé)

1492-1421 (numérique)

Découvrir la revue

Citer ce document

Collet, T. (1992). Lexique anglais-français des sous-systèmes des satellites de télécommunications. Meta, 37(3), 523-537. https://doi.org/10.7202/002349ar d'utilisation que vous pouvez consulter en ligne.

https://apropos.erudit.org/fr/usagers/politique-dutilisation/ 


\section{ÉTUDES TERMINOLOGIQUES ET LINGUISTIQUES}

\section{LEXIQUE ANGLAIS-FRANCCAIS DES SOUS-SYSTĖMES DES SATELLITES DE TÉLECOMMUNICATIONS}

Au cours de leurs trente années d'existence - le premier satellite relais actif, qui portait le nom de Telstar I, ne fut lancé qu'en 1962 - les satellites de télécommunications et leur électronique, ainsi que les stations terriennes, se sont considérablement perfectionnés. Bien que la technique des télécommunications spatiales se soit développée, au début, principalement aux États-Unis, la terminologie spatiale en langue française est, aujourd'hui, relativement bien établie. Il existe cependant très peu de dictionnaires techniques - nous n'en avons pas trouvé - qui se concentrent uniquement sur le satellite, ses sous-systèmes et les équipements à bord. Le présent Lexique anglais-français des sous-systèmes des satellites de télécommunications se propose donc de regrouper les principaux termes qui sont utilisés dans le domaine des télécommunications spatiales pour désigner les parties et les équipements d'un satellite de télécommunications quelconque.

Nous ne prétendons nullement fournir au lecteur un répertoire exhaustif des termes utilisés dans le domaine des télécommunications spatiales, étant donné qu'il s'agit d'un domaine très vaste. Souhaitons toutefois que ce lexique, fruit de nombreuses heures de travail et de recherches, puisse lui être utile.

\begin{tabular}{|c|c|}
\hline $\mathbf{A B M}$ & V. apogee boost motor \\
\hline accelerometer & accéléromètre m. \\
\hline adapter; separation adapter & adaptateur d'intégration $\mathrm{m}$. ; adaptateur $\mathrm{m}$. \\
\hline aerial; antenna & antenne $f$. \\
\hline AKM & V. apogee boost motor \\
\hline amplifier & amplificateur $\mathrm{m}$. \\
\hline antenna; aerial & antenne $\mathrm{f}$. \\
\hline $\begin{array}{l}\text { antenna dish; dish; } \\
\text { parabolic reflector }\end{array}$ & réflecteur parabolique $\mathrm{m}$. \\
\hline antenna feed; feed & source primaire $f$. \\
\hline antenna reflector; reflector & $\begin{array}{l}\text { réflecteur d'antenne } \mathbf{m} . \text {; réflecteur hertzien } \mathbf{m} \text {.; réflec- } \\
\text { teur } \mathrm{m} \text {. }\end{array}$ \\
\hline perture antenna & antenne à ouverture $\mathrm{f}$. \\
\hline ogee-boost motor & V. apogee boost motor \\
\hline ogee boost motor; & moteur d'apogée m. \\
\hline
\end{tabular}

apogee-boost motor; apogee kick motor; apogee motor; ABM; AKM 


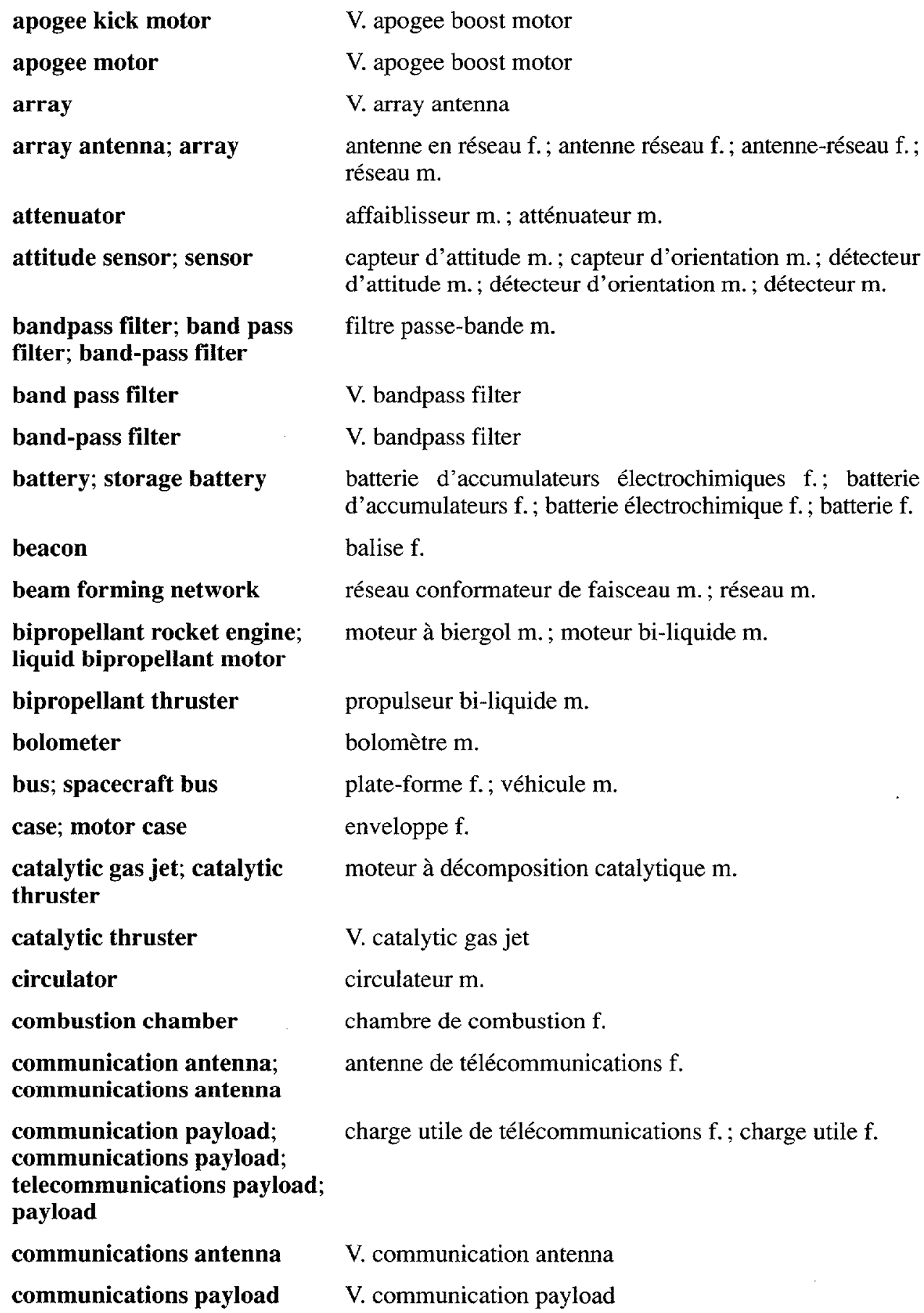


communications repeater; payload repeater; satellite repeater; repeater communications transponder; satellite transponder; spacecraft transponder; transponder

crystal-controlled oscillator; crystal oscillator; $\mathrm{XO}$

crystal oscillator

cylindrical panel cylindrical solar panel; cylindrical panel

deployable reflector; unfurlable reflector

despun antenna

dipole

dipole antenna; dipole

directional antenna

dish; parabolic reflector; antenna dish

dish antenna; parabolic reflector antenna; parabolic antenna

\section{double conversion} transponder; doublefirequency-conversion repeater

double-frequency-conversion $V$. double conversion transponder rrepeater

downconverter; downconverter

\section{down-converter}

drum

earth coverage antenna

earth-horizon sensor

earth horizon sensor; earth-horizon sensor; earth sensor panneau solaire cylindrique $\mathrm{m}$. répéteur de télécommunications $\mathrm{m}$.; répéteur de satellite $\mathrm{m}$. ; répéteur $\mathrm{m}$.

oscillateur à quartz m.

V. crystal-controlled oscillator

V. cylindrical solar panel

réflecteur déployable $\mathrm{m}$.

antenne contre-rotative f.

V. dipole antenna

antenne doublet $\mathrm{f}$; ; doublet $\mathrm{m}$.

antenne directive $\mathrm{f}$.

réflecteur parabolique $m$.

antenne à réflecteur parabolique f.; antenne parabolique f.

répéteur à double changement de fréquence $\mathrm{m}$.

changeur abaisseur de fréquence $\mathrm{m}$.; changeur inverseur $\mathrm{m}$. ; convertisseur abaisseur $\mathrm{m}$. ; mélangeur abaisseur de fréquence $m$.; mélangeur soustractif $m$.

V. downconverter

cylindre $\mathrm{m}$.

antenne à couverture globale f.; antenne à faisceau global f.; antenne globale $\mathrm{f}$.

V. earth horizon sensor

capteur terrestre $\mathbf{m}$.; détecteur d'horizon infrarouge terrestre $\mathrm{m}$.; détecteur d'horizon terrestre m.; détecteur d'horizon $\mathrm{m}$. ; détecteur terrestre $\mathrm{m}$. 
earth sensor

EDA; electronically despun antenna

electronically despun antenna; EDA

equipment panel

equipment shelf

feed; antenna feed

feed array

feedhorn; feed horn

feed horn

filter

flat panel; flat solar panel

flat solar panel

frequency synthesizer

GaAsFET amplifier; solid state amplifier; solid-state amplifier; solid-state power amplifier; SSPA

gyro

gyroscope; gyro

heat pipe

heat radiator; thermal radiator; radiator;

heat shield

heat shield

helical antenna;

helix antenna

helix antenna

hemi / zone antenna

high-gain antenna

high gain antenna;

high-gain antenna

high-power amplifier

high power amplifier; high-power amplifier; HPA
V. earth horizon sensor

antenne contre-rotative électronique $\mathrm{f}$

antenne contre-rotative électronique $\mathrm{f}$

panneau $\mathrm{m}$.

plateau $\mathrm{m}$.

source primaire $f$.

réseau de sources $\mathrm{m}$.

cornet primaire $\mathrm{m}$.

V. feedhorn

filtre $m$.

panneau solaire plan $\mathrm{m}$.

V. flat panel

synthétiseur de fréquence $m$.

amplificateur à arséniure de gallium à effet de champ m.; amplificateur à état solide m.; amplificateur de puissance à l'état solide $\mathrm{m}$.

V. gyroscope

gyroscope $\mathrm{m}$. ; gyro $\mathrm{m}$.

caloduc $\mathrm{m}$.

radiateur thermique $\mathrm{m}$.; radiateur $\mathrm{m}$.; bouclier thermique $\mathrm{m}$.

V. heat radiator

antenne en hélice f.; antenne hélice f.

V. helical antenna

antenne pour couverture d'hémisphères et de zones $f$.

$\mathrm{V}$. high gain antenna

antenne à grand gain $\mathrm{f}$.

V. high power amplifier

amplificateur à grande puissance $\mathbf{m}$. 
horn

horn antenna; horn

horn

HPA

hydrazine thruster

IF amplifier; i.f. amplifier; i-f amplifier; intermediate-frequency amplifier

i.f. amplifier
i-f amplifier
infrared earth sensor;
IR earth sensor;
infrared sensor; IR sensor
infrared sensor
input multiplexer

intermediate-frequency
amplifier
ion thruster
IR earth sensor
IR sensor
isolator
jet; orbit control thruster;
thruster

lens antenna

liquid bipropellant motor; bipropellant rocket engine

liquid engine

liquid-fuel engine

liquide-fuel rocket
V. horn antenna

antenne cornet $f$. ; antenne-cornet $f$. ; cornet $m$.

cornet $\mathbf{m}$.

V. high power amplifier

propulseur à hydrazine $\mathbf{m}$.

amplificateur à fréquence intermédiaire m.; amplificateur FI m.

\section{IF amplifier \\ V. IF amplifier}

détecteur terrestre infrarouge m.; détecteur infrarouge m.

V. infrared earth sensor

démultiplexeur d'entrée $\mathrm{m}$.; démultiplexeur $\mathrm{m}$.; DEMUX m.; filtre démultiplexeur d'entrée $\mathrm{m}$.; filtre démultiplexeur m. ; filtre multiplexeur d'entrée m.; multiplexeur d'entrée m.; MUXE m.

V. IF amplifier

propulseur ionique $\mathrm{m}$.

V. infrared earth sensor

V. infrared earth sensor

isolateur $\mathrm{m}$.

propulseur de commande d'orbite m.; propulseur de correction d'orbite $\mathrm{m}$.; propulseur de correction $\mathbf{m}$.; propulseur $m$.

antenne à lentille $\mathrm{f}$.

moteur à biergol m.; moteur bi-liquide $\mathrm{m}$.

V. liquid-fuel rocket engine

V. liquid-fuel rocket engine

V. liquid-fuel rocket engine 
liquid-fuel rocket engine; liquid-fuel engine; liquidfuel rocket; liquid-propellant rocket engine; liquidpropellant rocket motor; liquid-propellant engine; liquid-propellant rocket; liquid engine

liquid-propellant engine liquid-propellant rocket liquid-propellant rocket engine

liquid-propellant rocket motor

LNA; low-noise amplifier

LO; local oscillator

local oscillator; LO

low-noise amplifier; LNA

magic tee

master oscillator

mechanically despun antenna

mesh

microwave antenna

microwave filter

microwave switch matrix; MSM

mixer

MLI

momentum wheel

motor case ; case

MSM

multibeam antenna; multi-beam antenna; multiple beam antenna; multiple-beam antenna moteur à propergol liquide m.; moteur à propergols liquides $\mathrm{m}$.

\section{V. liquid-fuel rocket engine \\ V. liquid-fuel rocket engine \\ V. liquid-fuel rocket engine}

V. liquid-fuel rocket engine

amplificateur à faible bruit $\mathrm{m}$.; AFB

oscillateur local m.; OL

oscillateur local m.; OL

amplificateur à faible bruit m.; AFB

té magique $\mathrm{m}$.

maittre oscillateur m.; oscillateur pilote m.; pilote m.

antenne contre-rotative mécanique $f$.

tricot métallique souple m.; tricot métallique m.; tricot $\mathrm{m}$.; voile maillé souple $\mathrm{m}$.; voile métallique souple $\mathrm{m}$.; voile souple $m$.; voile $m$.

antenne hyperfréquence $f$.

filtre hyperfréquence $\mathrm{m}$.

matrice de commutation hyperfréquence $f$.

mélangeur $\mathbf{m}$.

V. multilayer insulation blanket

roue à inertie f.; roue cinétique f.; roue d'inertie f.; volant cinétique $\mathrm{m}$.; volant d'inertie $\mathrm{m}$.

enveloppe $\mathrm{f}$.

V. microwave switch matrix

antenne à faisceaux multiples $\mathrm{f}$.; antenne multifaisceau $\mathrm{f}$. 


\author{
multi-beam antenna \\ multilayer insulation \\ blanket; MLI

\section{multiple-beam antenna \\ multiple beam antenna; \\ multiplexer}

NiCad battery; nickelcadmium battery

nickel-cadmium battery

nickel-cadmium cell

nondirectional antenna;

non-directional antenna;

omnidirectional antenna;

omni antenna; omni-antenna

non-directional antenna

nozzle

nutation damper

omni antenna

omni-antenna

omnidirectional antenna

orbit control thruster;

thruster; jet

oscillator

output multiplexer

parabolic antenna; dish antenna; parabolic reflector antenna

parabolic reflector; dish; antenna dish

parabolic reflector antenna

payload

payload repeater

perigee kick motor; PKM
V. multibeam antenna

matelas de superisolation

multicouche m.; matelas multicouche de protection thermique $\mathrm{m}$.; superisolation multicouche $\mathrm{f}$.

V. multibeam antenna

V. multibeam antenna

filtre multiplexeur $\mathrm{m}$.; multiplexeur $\mathrm{m}$.

batterie au nickel-cadmium $\mathrm{f}$; batterie nickel-cadmium f. ; batterie $\mathrm{Ni}-\mathrm{Cd}$ f.

V. NiCad battery

accumulateur au nickel-cadmium m.; accumulateur nickel-cadmium $\mathrm{m}$.

antenne équidirective $\mathrm{f}$.

V. nondirectional antenna

tuyère $f$.

amortisseur de nutation $\mathrm{m}$.

V. nondirectional antenna

V. nondirectional antenna

V. nondirectional antenna

propulseur de commande d'orbite m.; propulseur de correction d'orbite $\mathrm{m}$.; propulseur de correction m.; propulseur $\mathrm{m}$.

oscillateur $\mathrm{m}$.

filtre multiplexeur de sortie m.; multiplexeur de sortie m.; MUX m.

antenne à réflecteur parabolique $\mathrm{f}_{\text {; }}$; antenne parabolique $f$.

réflecteur parabolique $\mathbf{m}$.

V. parabolic antenna

$\mathrm{V}$. communication payload

V. communications repeater

moteur de périgée $m$. 


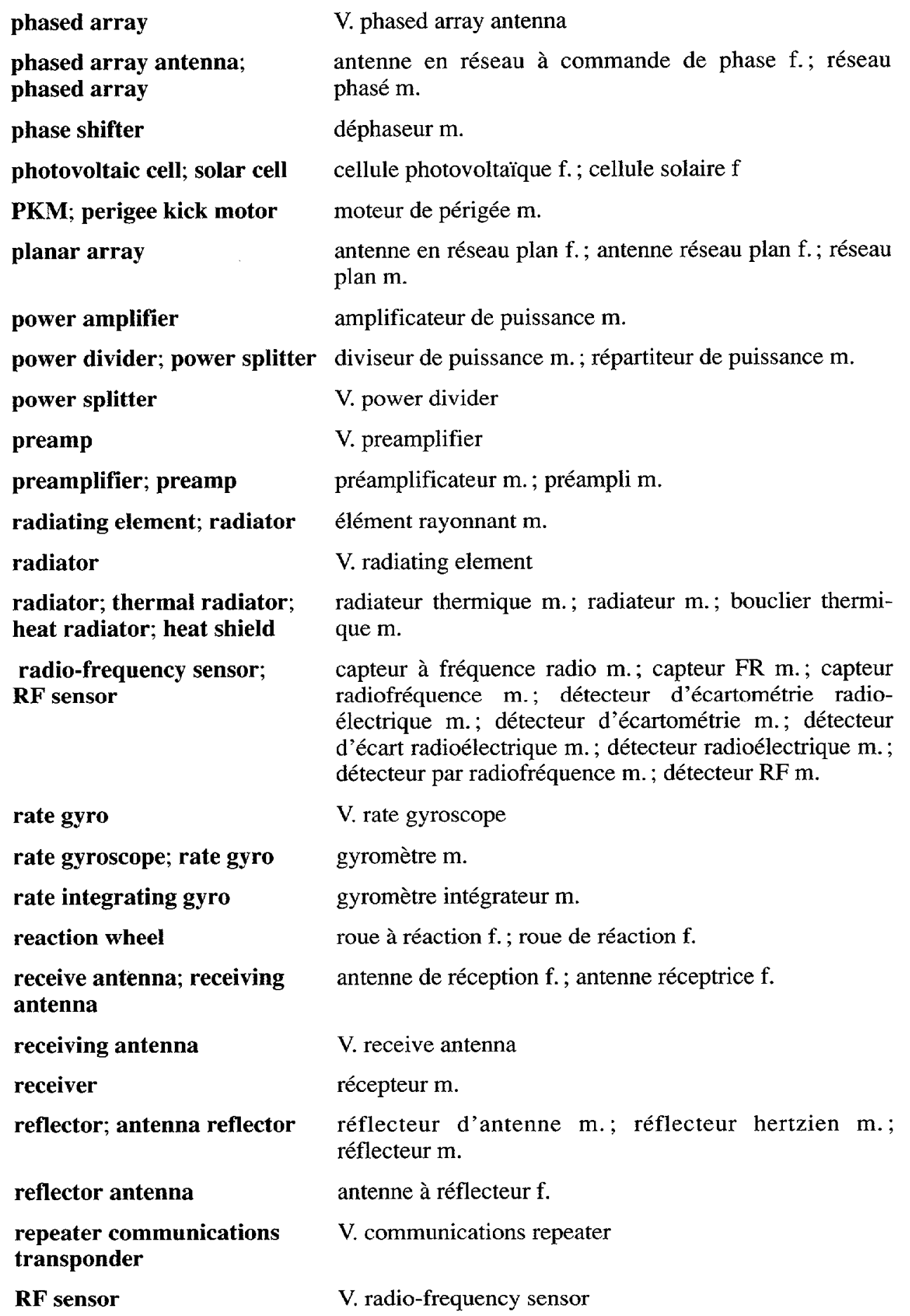




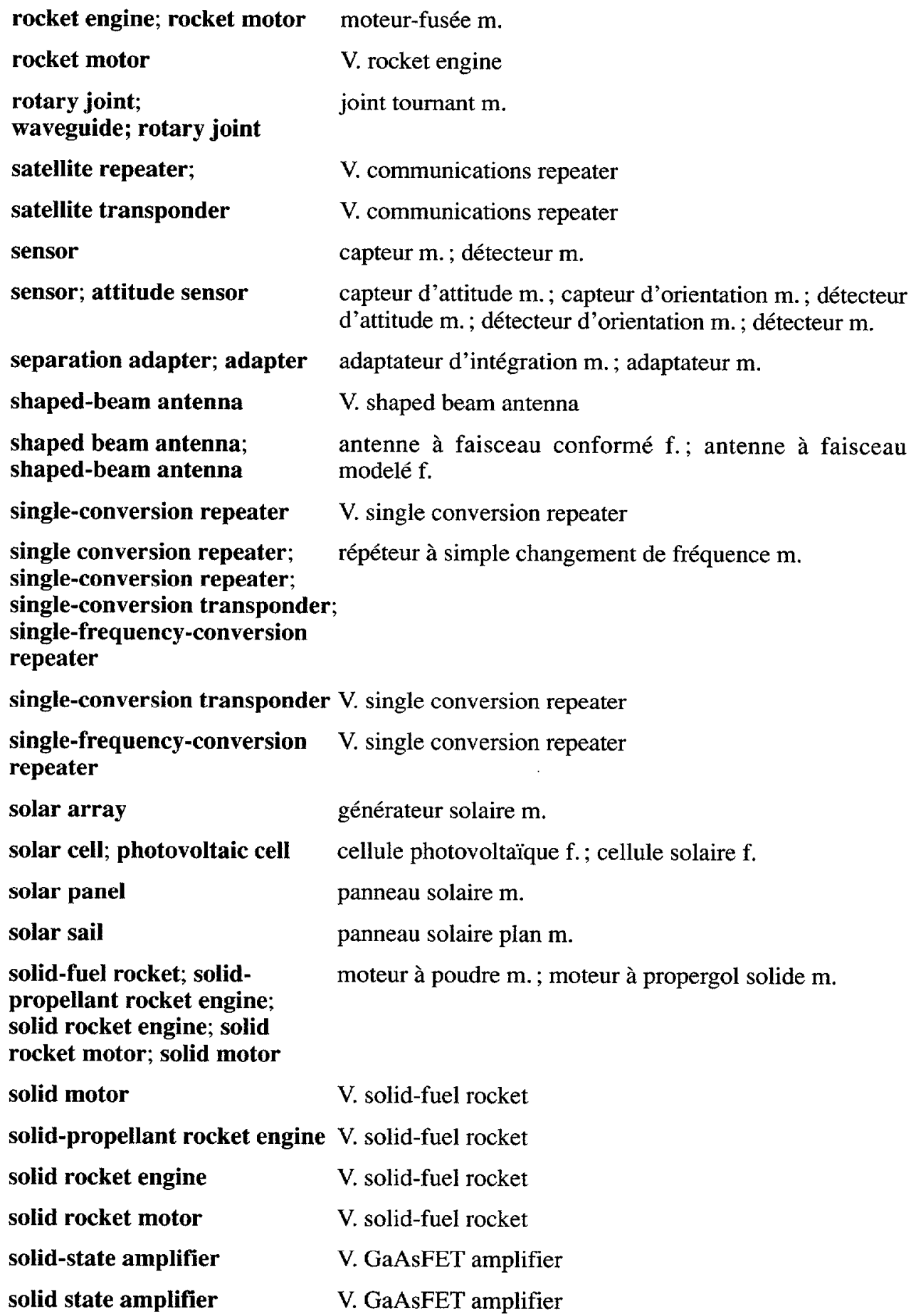




solid-state power amplifier
spacecraft bus; bus
spacecraft transponder
spot-beam antenna
spot beam antenna;
spot-beam antenna
SSPA

SSPA

star sensor

storage battery; battery

sun sensor

superinsulated blanket; superinsulation thermal blanket; superinsulation blanket

superinsulation blanket

superinsulation thermal blanket

switch matrix

tank

telecommunications payload communications payload; communication payload; payload

TDA; tunnel diode amplifier telemetry and command antenna; TT \& $\mathrm{C}$ antenna

thermal radiator;

heat radiator; radiator; heat shield

thruster

transistor amplifier

transistor

transmit antenna; transmitting antenna

transmitter

transmitting antenna
V. GaAsFET amplifier

plate-forme f.; véhicule m.

V. communications repeater

V. spot beam antenna

antenne à faisceau étroit $\mathrm{f}$. ; antenne à faisceau fin $\mathrm{f}$.

\section{GaAsFET amplifier}

détecteur d'étoile m.; détecteur stellaire m.

batterie d'accumulateurs électrochimiques f.; batterie d'accumulateurs f.; batterie électrochimique f.; batterie f.

capteur solaire m.; détecteur solaire m.

matelas de superisolation $\mathrm{m}$.; revêtement superisolant $\mathrm{m}$. ; superisolation $\mathrm{f}$.

V. superinsulated blanket

V. superinsulated blanket

matrice de commutation $\mathrm{f}$.

réservoir $\mathrm{m}$.

charge utile de télécommunications f.; charge utile f.

amplificateur à diode tunnel $\mathrm{m}$.

antenne de télémesure et de télécommande f.; antenne de télémesure - télécommande f.

radiateur thermique $\mathrm{m}$.; radiateur $\mathrm{m}$.; bouclier thermique

V. orbit control thruster

amplificateur à transistor m.; amplificateur à transistors m.

transistor $\mathrm{m}$.

antenne d'émission f.;

antenne émettrice f.

émetteur $\mathrm{m}$.

V. transmit antenna 
transponder

traveling-wave tube

traveling wave tube; traveling-wave tube; $T W T$

traveling-wave-tube amplifier

traveling wave tube amplifier; traveling-wavetube amplifier; TWT amplifier; TWTA

triplexer

TT \& C antenna

tunnel diode amplifier; TDA

TWT

TWTA

TWT amplifier

unfurlable reflector; deployable reflector

upconverter; up-converter

up-converter

waveguide; wave guide

wave guide

waveguide rotary joint; rotary joint

wideband receiver; wide-band receiver

wide-band receiver

XO
V. communications repeater

V. traveling wave tube

tube à onde progressive $\mathrm{m}$. ;

tube à ondes progressives m. ; TOP; T.O.P.

$\mathrm{V}$. traveling wave tube amplifier

amplificateur à tube à onde progressive m.; amplificateur à tube à ondes progressives $\mathrm{m}$.; amplificateur à TOP m.; amplificateur TOP m. ; ATOP

triplexeur $\mathrm{m}$.

$\mathrm{V}$. telemetry and command antenna

amplificateur à diode tunnel $\mathrm{m}$.

V. traveling wave tube

V. traveling wave tube amplifier

V. traveling wave tube amplifier

réflecteur déployable $\mathrm{m}$.

changeur élévateur de fréquence $m$.; changeur non inverseur $\mathrm{m}$.; convertisseur élévateur $\mathrm{m}$.; mélangeur additif $\mathrm{m}$.; mélangeur élévateur de fréquence $\mathrm{m}$.

V. upconverter

guide d'onde $\mathrm{m}$. ; guide d'ondes $\mathrm{m}$.

V. waveguide

joint tournant $\mathrm{m}$.

récepteur à large bande $\mathrm{m}$.

$\mathrm{V}$. wideband receiver

V. crystal-controlled oscillator

ABADIE, Lucien (1971): «Les satellites de télécommunications», L'aéronautique et l'astronautique, no 27 , pp. 59-68.

ABADIE, Lucien (1987) : «Les programmes de satellites en cours à l'Aérospatiale», L'aéronautique et l'astronautique $\mathrm{n}^{\text {os }} 123-124$, pp. 69-73. 
ABUTALEB, G. E. A. (1977): «The COMSTAR Satellite System», COMSAT Technical Review, vol. 7, no 1 , pp. 35-83.

ALLEN, William H. (Ed.) (1965): Dictionary of Technical Terms for Aerospace Use, Washington, D. C., National Aeronautics and Space Administration (NASA), 314 p.

ANGELO, Joseph A. (1983): The Dictionary of Space Technology, New York, Van Nostrand Reinhold Company, $380 \mathrm{p}$.

ARMIN, R. et J.-P. BAUDRY (1989) : «TDF/TV-SAT, satellites français et allemand de télédiffusion directe», L'aéronautique et l'astronautique, $\mathrm{n}^{\circ} 135$, pp. 42-49.

ASSAL, F. et al. (1982): «Satellite Switching Center for SS-TDMA Communications», COMSAT Technical Review, vol. $12, \mathrm{n}^{\circ} 1$, pp. 29-68.

Association française de normalisation (1960) : Vocabulaire électrotechnique - Groupe 50-Électrochimie et électrométallurgie (NF C01 - 050), Paris La Défense, AFNOR, mai 1960, 31 p.

Association Française de Normalisation (1972): Vocabulaire électrotechnique - Groupe 62 - Antennes (NF C01 - 062), Paris, La Défense, AFNOR, février 1972, $33 \mathrm{p}$.

BARGELLINI, P. L. (1972): «The Intelsat IV Communications System», COMSAT Technical Review, vol. 2, nº 2, pp. 437-572.

BARKATS, G. (1979) : «Développement d'un générateur solaire rigide dépliable pour des satellites géostationnaires stabilisés 3 axes», L'aéronautique et l' astronautique, $\mathrm{n}^{\circ} 78, \mathrm{pp} .29-42$.

BARTHOLOMÉ, P. (1979): «Le programme européen OTS de satellite de télécommunications. Développement du satellite et performances en orbite», L'aéronautique et l'astronautique, $\mathrm{n}^{\circ} 75, \mathrm{pp} .3-14$.

BERNATEAU et al. (1968): «Antenne contre-rotative électronique à grande directivité», L'aéronautique et l'astronautique, no 6 , pp. 61-67.

BLEAZARD, G. B. (1985): Introducing Satellite Communications, Manchester (England), NCC Publications, $350 \mathrm{p}$.

BOSTIAN, Charles W. et Timothy PRATT (1986): Satellite Communications, New York, John Wiley \& Sons, $472 \mathrm{p}$.

BOUSSO, Jacques (1985) : «Amplificateurs. Généralités et caractérisation», Techniques de l'ingénieur, Électronique générale. Circuits E 3 II, pp. E 3300-1, E 3300-10.

BRILLAUD, Jean-Paul (1988): «Télécommunications par satellite», Techniques de l'ingénieur, Télécommunications E 7, pp. E 7570-1, E 7570-11.

Bureau de normalisation du Québec (1975): Vocabulaire électrotechnique. Groupe 63. Technique radioélectrique générale. Antennes, émetteurs et récepteurs radioélectriques, $\mathrm{BNQ}, 71 \mathrm{p}$

BURGIO, M. (1989) : «Le programme Eutelsat II», L'aéronautique et l'astronautique, no 135, pp. 50-53.

CAMPANELLA, S. J. et al. (1986): «Advantages of TDMA and Satellite-Switched TDMA in Intelsat V and VI», COMSAT Technical Review, vol. 16, n० 1, pp. 207-238.

CANNON, Don L. et Gerald LUECKE (1980): Understanding Communications Systems, Dallas, Texas Instruments, pp. 1-1, 10-30.

CARTER, Harley (1963): Dictionary of Electronics, 2nd ed., London, George Newnes Limited, $410 \mathrm{p}$.

Centre national d'études spatiales (CNES) et Centre national d'études des télécommunications (CNET) (1982): Télécommunications spatiales. I. Bases théoriques, Paris, Masson, $412 \mathrm{p}$.

Centre national d'études spatiales (CNES) et Centre national d'études des télécommunications (CNET) (1983): Télécommunications spatiales. II. Secteur spatial, Paris, Masson, $386 \mathrm{p}$.

Centre national d'études spatiales (CNES) et Centre national d'études des télécommunications (CNET) (1983): Télécommunications spatiales. III. Secteur terrien. Systèmes de télécommunications par satellite, Paris, Masson, $451 \mathrm{p}$

Centre national d'études spatiales (CNES) et Conseil international de la langue francaise (CILF) (1985): Dictionnaire de spatiologie. Sciences et techniques spatiales. Tome 1. Termes et définitions, $2^{\mathrm{e}}$ éd., Paris, CILF, $350 \mathrm{p}$

Centre national d'études spatiales (CNES) et Conseil international de la langue française (CILF) (1986): Dictionnaire de spatiologie. Sciences et techniques spatiales. Tome 2. Traductions anglais - allemand espagnol-russe, $2^{\mathrm{e}}$ éd., Paris, CILF, $536 \mathrm{p}$.

COHEN, P. J. et al. (1988): Inventaire des compétences de recherche du milieu universitaire québécois en matière de communications par satellites, Montréal, Centre de développement technologique de l'École Polytechnique de Montréal, pp. 3-69.

COMBES, Paul F., J. GRAFFEUIL et J.-F. SAUTEREAU (1985) : Composants, dispositifs et circuits actifs en micro-ondes, Paris, Bordas et Dunod, $239 \mathrm{p}$.

COMBES, Paul-François (1986) : «Antennes pour techniques spatiales», Techniques de l'ingénieur, Télécommunications E 7, pp. E 7590-1, E 7590-21.

Commission électrotechnique intemationale (CEI) (1970): Vocabulaire Électrotechnique International, Groupe 60. Radiocommunications, $2^{\mathrm{e}}$ éd., Genève, Bureau central de la Commission électrotechnique internationale $275 \mathrm{p}$. 
Commission électrotechnique internationale (CEI) (1982): Édition anticipée du Vocabulaire électrotechnique international, chapitre 725, «Radiocommunications spatiales», Genève, Bureau central de la Commission électrotechnique internationale, $32 \mathrm{p}$.

Commission Électrotechnique Internationale (CEI) (1982): Vocabulaire électrotechnique international, chapitre 726, «Lignes de transmission et guides d'ondes», Genève, Bureau central de la Commission électrotechnique internationale, $136 \mathrm{p}$.

Commission Électrotechnique Internationale (CEI) (1983): Dictionnaire CEl multilingue de l'électricité. Volume 1, Genève, Bureau central de la Commission électrotechnique internationale, $889 \mathrm{p}$.

Commission Électrotechnique Internationale (CEI) (1984): Vocabulaire électrotechnique international, chapitre 521, «Dispositifs à semiconducteurs et circuits intégrés», Genève, Bureau central de la Commission électrotechnique internationale, $90 \mathrm{p}$.

CONSIDINE, Douglas M. (éd.) (1989): Van Nostrand's Scientific Encyclopedia, 7th ed., New York, Van Nostrand Reinhold Company, $3180 \mathrm{p}$.

CURTIN, Denis J. (éd.) (1979): Trends in Communications Satellites, Oxford, Pergamon Press, pp. 131-472.

DE BAYSER, P. et al. (1989): «Les futures missions de télécommunications européennes et les charges utiles associées", L'aéronautique et l'astronautique, $\mathrm{n}^{\circ} 135$, pp. 31-41.

DE LUCA, Johanne (1988): Dictionnaire anglais-français des télécommunications, Paris, Masson, 401 p.

DERIEUX, C. (1981): «TDF 1, satellite français de radiodiffusion», L'aéronautique et l'astronautique, no 91 , pp. 31-37.

DUIGOU, Michel (1977) : «Le satellite Intelsat $V »$, L'aéronautique et l'astronautique, $\mathrm{n}^{\circ} 62, \mathrm{pp} .3-10$.

DUMONT, Patrick et al. (1985): «Analyse du rayonnement des antennes à réflecteur déployable», Annales des télécommunications, vol. 40, nos 7-8, pp. 387-395.

DUREUX, Bernard (1979) : «Synthétiseurs de fréquence», Techniques de l'ingénieur, Électronique générale. Circuits E 3 II, pp. E 3870-1, E 3870-15.

ELBERT, Bruce R. (1987): Introduction to Satellite Communication, Boston, London, Artech House, 369 p.

Encyclopédie des sciences industrielles Quillet (1973-1974) : Paris, Librairie Aristide Quillet, 4 vol.

FEHER, Kamilo (éd.) (1983): Conference Proceedings. First Canadian Domestic and International Satellite Communications Conference, Amsterdam, North-Holland, pp. 1.1.1, 30.6.4.

FITAMANT, H. (1971) : «L'alimentation électrique des satellites», L'aéronautique et l'astronautique, no 31 , pp. 19-30.

FONTOLLIET, Pierre-Gérard (1983): Systèmes de télécommunications, Saint-Saphorin (Suisse), Éditions Georgi, $511 \mathrm{p}$.

GAGLIARDI, Robert M. (1984): Satellite Communications, Belmont (California), Lifetime Learning Publications, $474 \mathrm{p}$.

GARAGABEDIAN, Eugénie et Andy LAURISTON (1985): «Attitude and Orbit Control of Geostationary Satellites», Termiglobe, vol. VII, nos 4-5, pp. 19-34.

GARDIOL, Fred (1981): Hyperfréquences, Saint-Saphorin (Suisse), Éditions Georgi, 427 p.

GATLAND, K. W. (1964): Telecommunication Satellites, Englewood Cliffs (N.J.), Prentice-Hall, 441 p.

GRAF, Rudolf F. (1977): Modern Dictionary of Electronics, 5th ed., Indianapolis, Howard W. Sams \& Co., 832 p.

GRAHAM, John (1983): The Facts on File Dictionary of Telecommunications, New York, Facts on File Publications, $199 \mathrm{p}$.

GRÉGOIRE, J.-P. et C. BRAZZINI (1985) : «Structure de satellites. Principes de conception, technologies, matériaux», L'aéronautique et l'astronautique, $\mathrm{n}^{\circ} 111$, pp. 19-27.

HATCHER, Norman M. (1967): A Survey of Attitude Sensors for Spacecraft, Washington, D.C., National Aeronautics and Space Administration (NASA), $18 \mathrm{p}$.

HOLME, Stephen C. et al. (1988): «A Compact Seven-Way Power Divider for Satellite Beam Forming Networks», IEEE MTT-S Digest, New York, Institute of Electrical and Electronics Engineers (IEEE), pp. 665-668.

HOUSSIN, Jean-Pierre (1974) : «Les télécommunications spatiales», La Recherche, vol. 5, n० 42, pp. 130-138.

Institute of Electrical and Electronics Engineers (1973): IEEE Standard Definitions of Terms for Antennas, New York, IEEE, $18 \mathrm{p}$.

Institute of Electrical and Electronics Engineers (1983): IEEE Standard Definitions of Terms for Antennas, New York, IEEE, $29 \mathrm{p}$.

JAY, Frank (éd.) (1988): IEEE Standard Dictionary of Electrical and Electronics Terms, New York, Institute of Electrical and Electronics Engineers, $1270 \mathrm{p}$.

JILG, E. T. (1972): «The Intelsat IV Spacecraft», COMSAT Technical Review, vol. 2, no 2, pp. 271-389.

JOLY, Hubert, rédacteur en chef (1986) : Dictionnaire des industries, Paris, Conseil international de la langue française (CILF), $1082 \mathrm{p}$.

KAPLAN, Marshall H. (1976): Modern Spacecraft Dynamics \& Control, New York, John Wiley \& Sons, 415 p.

KEYES, L. (1989): Communications Payload, Sainte-Anne-de-Bellevue (Canada), Spar Aerospace Limited, s. p.

LACOMBE, J.-L. et al. (1978) : «Systèmes de contrôle d'attitude et d'orbite de satellites : évolution jusqu'aux années 80-90", L'aéronautique et l'astronautique, no 69, pp. 33-56. 
LAGOWSKI, R. G. (1977): «Attitude Determination for the ANIK Satellites», Canadian Aeronautics and Space Journal, vol. 23, no 2, pp. 77-87.

LAPIERRE, H. (1989): Structure Subsystem, Sainte-Anne-de-Bellevue (Canada), Spar Aerospace Limited, sp.

LARROQUE, Joël et al. (1988): «Étude et réalisation de modulateurs et de démodulateurs directs quadriphases pour la régénération à bord des satellites relais», Annales des télécommunications, vol. 43, nos 5-6, pp. 299-305.

LASALLE, M. (1970) : «Projet Dioscures. Les satellites et leur renouvellement», L'aéronautique et l'astronautique, $\mathrm{n}^{\mathrm{O}} 22$, pp. $20-29$

LAURISTON, Andy et Jocelyne LE NÉAL (1985): Dictionnaire bilingue des télécommunications internationales, vol. 2: Equipements de transmission, Montréal, Teleglobe Canada, 506 p.

Le grand atlas de l' espace (1987): Paris, Encyclopædia Universalis France S.A., 397 p.

L'énergie photovoltaique et ses applications dans l'espace et sur terre. The Photovoltaic Power and its Applications in Space and on Earth (1973): Bretigny-sur-Orge (France), Centre national d'études spatiales (CNES), $679 \mathrm{p}$.

«Le satellite expérimental de télécommunications tactiques TACSAT 1» (1971): L'aéronautique et l'astronautique, $\mathrm{n}^{\circ} 27$, pp. 71-77.

LIPKE, D. W. et al. (1977): «MARISAT. A Maritime Satellite Communications System», COMSAT Technical Review, vol. 7, no 2, pp. 351-391.

LOMBARD, Didier et al. (1982) : «Système Télécom 1», Techniques de l'ingénieur, Télécommunications E 7 , pp. E 7565-1, E 7565-12.

LUKASHOK, Alvin (1967): Communication Satellites. How They Work, New York, G. P. Putnam's Sons, 160 p.

MANUALI, Bertrand (1967) : L'exploration spatiale et ses techniques. Trajectoires, fusées, satellites, Paris, Hermann, $216 \mathrm{p}$

MARAL, G., M. BOUSQUET et J. PARES (1982) : Les systèmes de télécommunications par satellites, Paris, Masson, $278 \mathrm{p}$.

MARKUS, John (1978): Electronics Dictionary, 4th ed., New York, McGraw-Hill Book Company, 744 p.

MARTIN, E. R. (1981): «Satellite Characteristics», COMSAT Technical Review, vol. 11, no 2, pp. 227-240.

MARTIN, James (1977): Future Developments in Telecommunications, 2nd ed., Englewood Cliffs (N.J.), Prentice-Hall, $668 \mathrm{p}$.

MARTIN, James (1978): Communications Satellite Systems, Englewood Cliffs (N.J.), Prentice-Hall, 398 p.

Meeting on Skynet (1970): The Institution of Electrical Engineers (IEE), 238 p.

MEYERS, Robert A. (éd.) (1989): Encyclopedia of Telecommunications, San Diego, Academic Press, 575 p.

MITTRA, R., W. A. IMBRIALE et E. J. MAANDERS (éd.) (1983): Satellite Communication Antenna Technology, Amsterdam, North-Holland, $652 \mathrm{p}$.

MORVAN, R. G. (dir.) (1969-1973) : Encyclopédie internationale des sciences et des techniques, France, Les Presses de la Cité, 10 vol.

NAYLER, J. L. (1964): A Dictionary of Astronautics, New York, Hart Publishing Company, 316 p.

NEILSON, Laura (1990): «Communications Satellites», Termiglobe, vol. XIII, no 2, pp. 1-4.

NEYRET, Pierre (1985): «Antenna Technology at INTELSAT», Annales des Télécommunications, vol. 40, nos $7-8$, pp. 361-377.

NOUVEL, Marcel (1983) : Dictionnaire bilingue des télécommunications internationales, vol. 1, Théorie des antennes, Montréal, Teleglobe Canada, $128 \mathrm{p}$.

PAIRITZ, Herbert A. (1985): Telecommunications Digest, New York, McGraw-Hill Book Company, 270 p.

PARES, J. et V. TOSCER (1975): Les systèmes de télécommunications par satellites, Paris, Masson, $221 \mathrm{p}$.

PARKER, D. (1989): Power Subsystem, Sainte-Anne-de-Bellevue (Canada), Spar Aerospace Limited, s. p.

PARKER, Sybil P. (éd.) (1984): McGraw-Hill Concise Encyclopedia of Science \& Technology, New York, McGraw-Hill Book Company, 2065 p.

PARKER, Sybil P. (éd.) (1984): McGraw-Hill Dictionary of Science and Engineering, New York, McGraw-Hill Book Company, 942 p.

PARKER, Sybil P. (éd.) (1984): McGraw-Hill Dictionary of Scientific and Technical Terms, 3rd ed., New York, McGraw-Hill Book Company, $1781 \mathrm{p}$.

PARKER, Sybil P. (éd.) (1987): McGraw-Hill Encyclopedia of Science \& Technology, 6th ed., New York, McGraw-Hill Book Company, $20 \mathrm{v}$.

PHAM TAT, Dat (1985): «Radiodiffusion par satellite», Techniques de l'ingénieur, Radioélectricité E 6, pp. E 6430-1, E 6430-11.

PINDER, Richard et Robert ROUSSELOT (1971) : «Techniques spatiales», La banque des mots, no 1, pp. 103-109.

PORTER, Richard W. (1977): The Versatile Satellite, Oxford, Oxford University Press, 173 p.

PRITCHARD, WILBUR L. et Joscph A. SCIULLI (1986): Satellite Communication Systems Engineering, Englewood Cliffs (N.J.), Prentice-Hall, 399 p.

REYNAUD, P. (1974) : «Stabilisation des satellites: l'amortisseur de mutation», L'aéronautique et l'astronautique, $\mathrm{n}^{\circ} 44$, pp. $33-38$.

RICHARD, C. (dir.) (1984): Bulletin signalétique des télécommunications, tome 39, no 2, pp. 117-214. 
ROEDERER, A. G. (1984): «Antennes embarquées à réflecteurs multisources», Annales des télécommunications, vol. 39 , nos $1-2$, pp. 34-43.

ROSENBERG, Sanders D. (1982): «Liquid Bipropellant Engines for Large Telecommunications Satellites», Acta Astronautica, vol. 9, no 11, pp. 663-669.

ROSNER, Roy Daniel (1984): Satellites, Packets and Distributed Telecommunications, Belmont (California), Lifetime Learning Publications, $628 \mathrm{p}$.

RUEL, C. (1989): Thermal Control Subsystem, Sainte-Anne-de-Bellevue (Canada), Spar Aerospace Limited, s. p.

SANFOURCHE, J.-P. (1970) : «Étude et réalisation d'un détecteur stellaire», L'aéronautique et l'astronautique, no 19 , pp. 31-41.

SHARPE, Mitchell R. (1970): Satellites and Probes. The Development of Unmanned Space Flight, Garden City (New York), Doubleday \& Company, $192 \mathrm{p}$.

SIMONS, Rainee (1990): Optical Control of Microwave Devices, Boston, London, Artech House, $236 \mathrm{p}$.

SLATER, J. N. et L. A. TRINOGGA (1985): Satellite Broadcasting Systems. Planning and Design, Chichester (England), Ellis Horwood Limited, $166 \mathrm{p}$.

SMITH, Emerson C. (1971): Glossary of Communications, Chicago, Telephony Publishing Corp., 547 p.

SMITH, G. (1980): Storage Batteries, 3rd ed., London, Pitman Advanced Publishing Program, 234 p.

SOARES, R., J. OBREGON, J. GRAFFEUIL et al. (1984) : Applications des transistors à effet de champ en arséniure de gallium, Paris, Eyrolles, $517 \mathrm{p}$.

SODEIKAT, Heinz (1974): «Charge utile de télécommunications du satellite Symphonie», L'aéronautique et l'astronautique, $\mathrm{n}^{\circ} 44, \mathrm{pp} .39-44$.

SPRING, K. (1989): Attitude Control Subsystem, Sainte-Anne-de-Bellevue (Canada), Spar Aerospace Limited, s. p.

The New Encyclopadia Britannica, (1984): 15th ed., Chicago, Encyclopædia Britannica Inc., 30 vol.

TURNER, Rufus P. et Stan GIBILISCO (1988): The Illustrated Dictionary of Electronics, 4th ed., Blue Ridge Summit (USA). TAB Books Inc., $648 \mathrm{p}$.

VAN TREES, Harry L. (éd.) (1979): Satellite Communications, New York, IEEE Press, 665 p.

VIDAL SAINT-ANDRÉ, Bruno et al. (1984): «Source primaire multi-éléments et matrice de commutation associée pour satellite de télécommunications utilisé en mode AMRT», Annales des télécommunications, vol. 39, nos 1-2, pp. 76-83.

«Vocabulaire aérospatial», (1973): L'aéronautique et l'astronautique, no 41, pp. 77-81.

VOULELIKAS, G. (1989): Propulsion Subsystem, Ste-Anne-de-Bellevue (Canada), Spar Aerospace Limited, s.p.

WALES, Robert O. (éd.) (1981): ATS - 6 Final Engineering Performance Report, Vol. I, Program and Systems Summaries; Mechanical and Thermal Details, Washington, D.C., National Aeronautics and Space Administration (NASA), $245 \mathrm{p}$.

WALES, Robert O. (éd.) (1981): ATS - 6 Final Engineering Performance Report, Vol. II, Orbit and Attitude Controls, Washington, D.C., National Aeronautics and Space Administration (NASA), 288 p.

WALES, Robert O. (éd.) (1981): ATS - 6 Final Engineering Performance Report. Volume III - Telecommunications and Power, Washington, D.C., National Aeronautics and Space Administration (NASA), $298 \mathrm{p}$.

WALKER, Peter M. B. (éd.) (1988): Chambers Science and Technology Dictionary, Cambridge, Edinburgh, W \& R Chambers Ltd., Cambridge University Press, 1008 p.

WEARMOUTH, C. et J. SUSPLUGAS (1975) : «La plate-forme OTS», L'aéronautique et l'astronautique, n 54 , pp. 17-26.

WELTI, G. (1971) : «La technologie des télécommunications par satellite dans la prochaine décennie», L'Onde électrique, vol. $51, \mathrm{n}^{\circ} 6, \mathrm{pp} .444-448$.

ZILIANI, M. A. (1986) : «Les générateurs solaires photovoltaïques du satellite à la station spatiale», L'aéronautique et l'astronautique, $\mathrm{n}^{\circ} 118$, pp. 15-20. 